its cash ends up at universities, although some are adept at winning funds: the University of Sheffield, which has strengths in engineering and manufacturing research, has received $£ 150$ million, for instance.

Outside Britain, the name Innovate UK is familiar to scientists and business people, but few know what it does, says Dan Breznitz, a researcher in innovation policy at the University of Toronto, Canada. One of the agency's major outputs is more widely appreciated, Breznitz says - its Catapult centres. This network of physical centres, which are loosely based on the Fraunhofer Institute's centres in Germany, provides equipment and resources to bridge the gap between university research and commercial technologies.

The largest, with $£ 150$ million in Innovate UK funding, is the Cell and Gene Therapy Catapult in London. It has "galvanized" UK researchers in the field, says Stuart Forbes, who

"The fund is going to be too big for academics to ignore." \section{works on regenera-} tive medicine at the University of Edinburgh. He relied on its advice to help get a potential cell therapy for liver cirrhosis into phase II trials. A first formal evaluation of the success of the Catapult centres - most of which are now five years old — will be published in September.

As Innovate UK's influence grows, it is in danger of losing focus on its core mission, says
Breznitz. If it now tries to act both like DARPA, with calls to solve key challenges, and like the US Small Business Innovation Research programme, which hands out grants to small research firms, it will struggle, he says.

The agency may also sharpen UK science's focus on economic returns. It uses economybased metrics, such as jobs generated and return on investment, to analyse its success, McKernan says.

She thinks that under UKRI, research councils might learn from this approach. "I would anticipate a much stronger analytics group across UKRI that understands how to get the return on investment from early, translational and applied research - and how we change some levers to get a stronger output," she says.

\title{
Giant radio telescope scaled back to contain costs
}

\section{Changes may affect the Square Kilometre Array's ability to observe the early Universe.}

\section{BY SARAH WILD}

$\mathrm{D}$ esigns for the world's largest radio telescope have been downgraded to save money - a decision that astronomers say could affect the instrument's ability to peer deep into the Universe's past.

The Square Kilometre Array (SKA), a telescope 50 times more sensitive than current instruments, is expected to cost billions of dollars. Its final design calls for around 2,000 radio dishes in Africa, together with up to 1 million antennas in Australia, with a total light-collecting area of roughly 1 square kilometre - hence the project's name.

But the first phase of construction, called SKA1, is a more modest affair. Already slimmed down from a larger design proposed in 2013, it now comprises 194 dishes in South Africa and around 130,000 antennas in Australia. In March, the SKA's board said that the project would have to find further cuts of around 20\% so that it could be built within a €674-million (US\$785-million) cap imposed by the project's ten funders - Australia, Canada, China, India, Italy, New Zealand, South Africa, Sweden, the Netherlands and the United Kingdom. And at a meeting in the Netherlands on 18-19 July, the board decided to make the savings by, among other measures, scaling back SKA1's computing power and crowding its antennas and radio dishes closer together.

It's the latter idea that concerns astronomers the most. Packing the telescope's individual components into a smaller space will mean a loss of resolution, making SKA1 less able to pick up on fine details. In most cases, this change won't seriously affect the array's scientific projects, says Tony Beasley, an astronomer and head of the US National Radio Astronomy Observatory in Charlottesville, Virginia. But Heino Falcke, an astronomer at Radboud
University in Nijmegen, the Netherlands, says that it may have an impact on the project's ability to detect faint signals emanating from a few hundred million years after the Big Bang, when the Universe's first stars and galaxies formed and began to emit light.

These low-frequency radio waves are to be picked up by the Australian antennas. Under the new cuts, clusters of these antennas known as low-frequency stations - will be placed a maximum of 40 kilometres apart, rather than 65 . With inferior resolution, the telescope could struggle to pick up lowfrequency signals over the noise of the Milky Way, says Falcke.

Astronomers were consulted about the changes at a meeting in Manchester, UK, in June. But since then, they have grown more concerned about the idea of crowding the Australian stations closer together. So the SKA board's decision may not be final: working

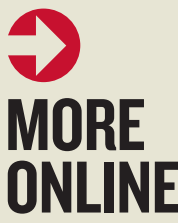

\section{TOP NEWS}

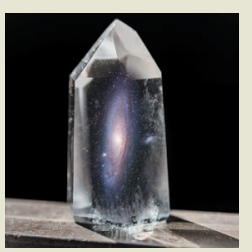

Big Bang gravitational effect observed in laboratory crystal go.nature. com/2tgpwse

\section{MORE NEWS}

- Australia cuts protections in marine parks go.nature.com/2uwnihe - Uganda trial shows it's worth paying to preserve trees go.nature.com/2v3ptr5 - Vaccines promoted as key to stamping out drug resistance go.nature.com/2typur6

\section{NATURE PODCAST}

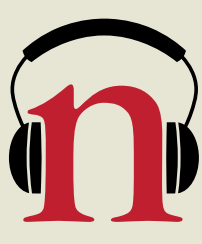

A brain-inspired computer; ageing control; and Al Gore the climate communicator nature.com/nature/ podcast 


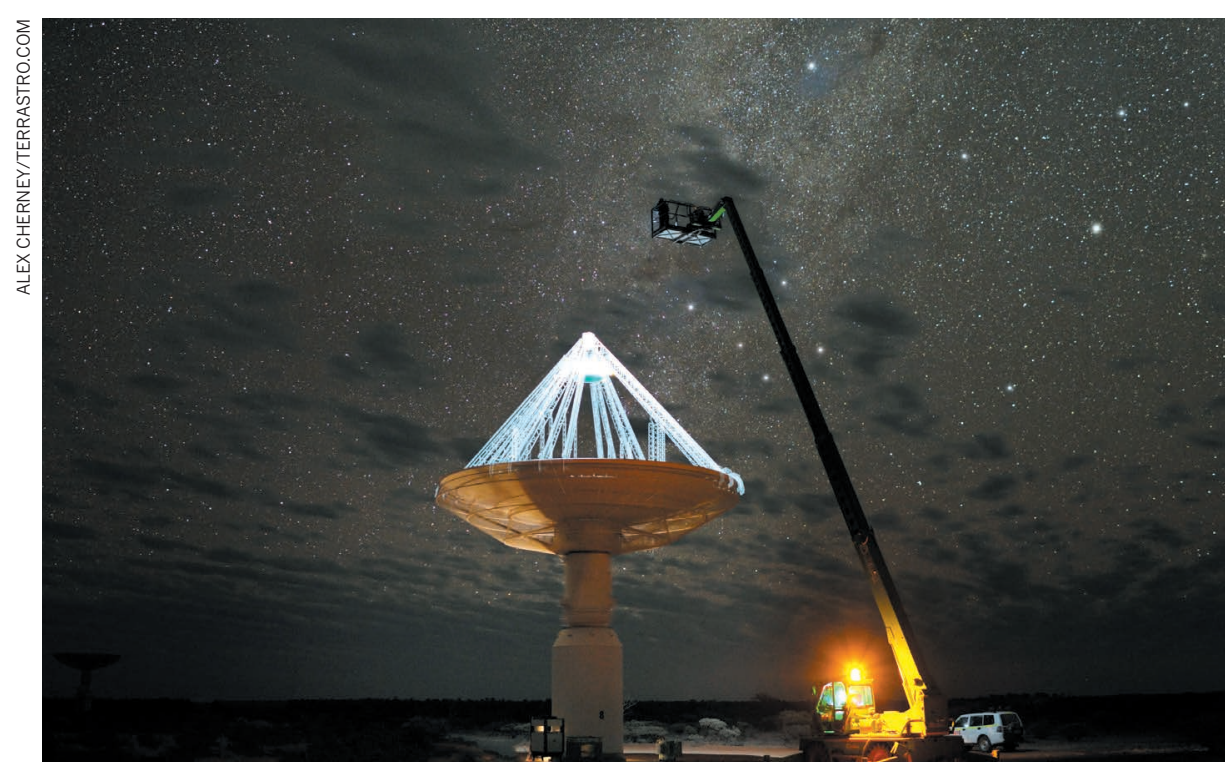

The Australian Square Kilometre Array (SKA) Pathfinder telescope is testing technology for the full SKA.

groups are still running simulations to work out the impact of the change, says Philip Diamond, the director-general of the SKA Organisation at the Jodrell Bank Observatory near Manchester.

Once construction starts on the antennas, it will be difficult and expensive to change the distances between them, says Roy Maartens, top of our list of things to restore once more funding becomes available."

Astronomers are less worried about the decrease in computing power, from 260 petaflops to 50 petaflops, says Falcke. Although this means it will take longer to process data, the limiting factor for translating SKAl's observations into images will be ironing out initial problems with software and algorithms. By the time those are sorted, the project will be looking to upgrade to a new generation of computers anyway, he says.

Diamond hopes that more countries can be persuaded to join the SKA project and inject extra funding, or that existing members will bring more money to the table. But some astronomers are not convinced that will happen. "In this environment, where they are continuously back-pedalling, are major partners going to come forward?" says one astronomer who asked not to be named.

Construction of SKA 1 is set to begin in mid-2019. But the SKA still lacks an intergovernmental organization to run it. Diamond says that a treaty to create such an organization should be ready this year, and all member countries will need to ratify it before construction starts. No funding has yet been agreed for developing the SKA beyond its first phase, but a construction push has been tentatively earmarked for the mid-2020s. 